\title{
Perspectives of Inter-professional Education Under a Global Infectious Menace
}

\author{
Jorge Cervantes $^{1}\left(\right.$ C) $\cdot$ Christiane Herber-Valdez $^{1,2}$
}

Accepted: 23 April 2021 / Published online: 28 April 2021

(c) International Association of Medical Science Educators 2021

\begin{abstract}
Health systems worldwide are facing unprecedented challenges, as a result of a convergence of major threats to our social and population health systems. For an epidemic of any magnitude, prevention and preparation by healthcare personnel in clinical settings are essential, both locally and globally. The need for the development of domestic and international training programs in the expanding field of emerging and reemerging infectious diseases is well recognized but particularly urgent at this time. Interprofessional education plays key roles in infectious diseases (ID) and in training the new generation of ID-related specialists.
\end{abstract}

Keywords Interprofessional education · Infectious disease $\cdot$ Global health

\section{Introduction}

Health systems worldwide are facing unprecedented challenges, as a result of a convergence of major threats to our social and population health systems. Inequities, violence, and lack of opportunity have led to major shifts in migration patterns within and across countries. At the same time, new infections, as well as behavioral and environmental hazards, threaten the health of all people [1]. The current COVID-19 outbreak has further compounded today's complex health issues, with devastating and unexpected impacts on our health systems and economies, requiring "unprecedented action and collaboration" across disciplines [2]. The coordination of efforts across healthcare professionals has never been as critical as it is today.

Jorge Cervantes

jorge.cervantes@ttuhsc.edu

1 Department of Medical Education, Paul L. Foster School of Medicine, Texas Tech University Health Sciences Center El Paso, El Paso, TX, USA

2 Office of the Provost and Vice President for Academic Affairs, Texas Tech University Health Sciences Center El Paso, El Paso, TX, USA

\section{New Demand and Opportunities for Inter-disciplinary Teams}

For an epidemic of any magnitude, prevention and preparation by healthcare personnel in clinical settings are essential, both locally and globally [3]. We have learned from previous outbreaks that it takes a team to contain and combat a virus epidemic [4], and the recent COVID-19 outbreak has taught us new lessons. During a pandemic, leaders must have the capacity to quickly organize multidisciplinary and interdisciplinary teams of professionals. These teams of professionals from various health-related fields, working together, are critical to both protecting society from microbial threats and in mounting effective responses to disease outbreaks [5].

The need to develop interdisciplinary healthcare teams is not a new concept. Interprofessional collaboration (IPC) emerged in healthcare several decades ago and has gained more support in the twenty-first century [6]. In 2001, a landmark publication by the Institute of Medicine (IOM) discussed the complexity of patients' needs, requiring the coordination of resources across a spectrum of settings, disciplines, and the community, stressing the need to develop collaborative teams to address these challenges through the sharing of expertise and resources [7]. There is wide agreement that today's patients require professionals from more than one healthcare discipline to address complex health issues [8], and collaborative interprofessional team environments have been shown to ensure optimal patient-centered care [9]. In 
addition, the World Health Organization (WHO) has linked IPC with better outcomes in family health, infectious disease, humanitarian efforts, responses to epidemics, and noncommunicable diseases [10]. Studies on collaborative care have shown improvements in access to healthcare and coordination of services, appropriate use of specialty care, chronic disease outcomes, and safety [10, 11]. Important indicators of safety, patient care, and environment of care, such as complications and error rates, length of hospital stay, conflict among caregivers, staff turnover, and mortality rates, have all been shown to decrease in collaborative care environments [10].

Despite these positive outcomes, evidence suggests that physicians are less inclined toward medical interdisciplinary teamwork than other health professionals [12], and while teamwork is practiced to some extent in today's healthcare settings, the question is whether it is recognized, promoted, and prioritized [1]. Most concerning, the lack of formal instruction and experience in multidisciplinary teamwork has been identified as a deficit in US physician education [13]. However, sometimes, the collaborative approach may face obstacles such as boundaries, time constraints, coordination of clinicians' availability, and the length of time taken for discussion [14].

\section{Interprofessional Education: Transforming Medical Learning}

Interprofessional education (IPE) occurs when students from two or more professions learn about, from, and with each other $[6,10]$. As summarized in the IOM's publication Interprofessional Education for Collaboration, interprofessional education provides opportunities to learn and practice skills that improve students' ability to communicate and collaborate, while also developing leadership qualities and respect for each other. As such, IPE prepares students for work in settings where collaboration is a key to success, as measured by "better and safer patient care as well as improved population health outcomes" [15].

In 2011, building on work by the World Health Organization (WHO), the Interprofessional Education Collaborative (IPEC) developed guiding definitions and competency domains for interprofessionalism, urging educators to develop curricular models to prepare health professions students to work together, with a common goal of building a safer and better patient-centered, population-oriented health care system $[16,17]$. According to Bridges et al. [8], IPE models help students better understand their own professional identity while also gaining understanding of other professionals" roles on the health care team, and "given the importance of quality care outcomes and the recognition that collaborative practice improves these outcomes, interprofessional education should be a high priority for every training institution".
Today, IPE is considered essential to medical education and training, as healthcare workers ought to be trained to work together as members of a "collaborative, practice-ready workforce" [10]. Exposing students to concepts and practices from a variety of health profession disciplines is widely considered a best practice and a vital curricular component of programs that train the next generation of health care professionals [1]. A systematic review of the literature demonstrated statistically significant positive impact and effectiveness of educational interventions with IPE programs in various healthcare disciplines [18]. In addition to improving team performance and patient safety in health care [19], IPE prepares medical students to work together with other health professionals once they enter careers in clinics or hospitals. IPE should start in pre-clerkship years and continue during clerkship [20] and residency training [21].

However, for learning to be truly interprofessional, it must include "purposeful integration and collaboration among the disciplines" [15]. Therefore, interprofessional collaborative education should include students representing nursing, medicine, dentistry, pharmacy and public health, as well as physical and occupational therapy, psychology, optometry, and social work. Institutions should commit to establishing true multi-professional healthcare communities, institutionalizing programs that aim to reduce barriers between disciplines, in order to help students and faculty better understand the respective underlying values of interdisciplinary systems and perspectives [5]. Given the recent convergence of threats that impact population health on a global scale, deliberate planning and configuration of specific disciplines for IPE are crucial for effective interprofessional training.

\section{Interprofessional Education in Infectious Diseases: Training the New Generation of ID Specialists}

The need for the development of domestic and international training programs in the expanding field of emerging and reemerging infectious diseases is well recognized [5], but particularly urgent at this time. Infectious diseases (IDs), in particular, require team work involving surveillance, immunization, containment, treatment, and interventions to modify social determinants [1].

Differences in skills and attitudes between specialist physicians can be gaping; however, research examining collaborative attitudes and preconceived inter-specialty stereotypes found that IPE programs in ID can result in increased perception of other specialties as collaborative [13]. The inclusion of pharmacists is particularly crucial to training in ID, as their role in antibiotic stewardships is critical in an era where the rate of antimicrobial development is outpacing work on antibiotic resistance [22]. For example, antimicrobial stewardship 
programs (ASPs) are essential components of health care systems and have become an area of focus for many hospitals around the world [9]. IPE on ASP improves knowledge and attitudes towards appropriate antimicrobial use and has been found to enhance collaboration between preclinical medical and pharmacy students [23]. In addition, a motivational faceto-face communication technique has been shown to be particularly useful in conveying ASP team member recommendations [9]. Although some institutions may not have a School of Pharmacy on their campuses, an option is to collaborate with a local or regional university that offers a pharmacy program.

\section{Interprofessional Education in a an Era of Globalization}

In addition to ensuring the representation of a variety of healthcare professions and emphasis on team-based care for improved healthcare outcomes, we also need to commit to creating a more diverse and international healthcare workforce. Not only is diversification necessary in this era of globalization, but it is crucial as patient populations are becoming increasingly diverse in terms of race, ethnicity, nationality, language, gender, religion, values, cultural, and socioeconomic backgrounds. Modern medical and healthcare education programs need to take active steps to provide opportunities and develop strategies to attract, retain, and prepare a diverse, global health care workforce to serve all populations.

Furthermore, training of healthcare professionals needs to be cognizant of the culture of medicine, including its lingo and values, which could be in stark contrast to those of patients. Scientific and technological literacy, as well as western-centric approaches to healthcare, which shape today's medical education, need to be acknowledged and critically evaluated. At the same time, new and effective approaches to health and disease need to be developed and incorporated into medical curricula. These new approaches will also need to address differences in support structures, resources, and educational modalities that operate in different population groups.

\section{Conclusion}

Professional education in medicine and allied health professions needs to keep pace with the challenges of health systems worldwide. As health education becomes increasingly globalized, standardization of knowledge, skill, and professionalism competencies will become necessary [1]. This would be a challenging goal at any time; however, the current convergence of events is adding both complexity and urgency. Changing population demographics and global infections are making it necessary to identify common denominators and to assess new approaches to healthcare across borders.
Teaching quality and transmission of knowledge, skills, and attitudes - with assessment of processes and outcomes, rather than content - will be the new paradigm to equip future physicians with the skills they will require to adapt and thrive within this new environment. In these changing times, where health burdens are shared across nations, single practitioner-based efforts do not suffice. An expanding body of literature suggests that interprofessional collaborative health care environments result in improved teamwork, patient outcomes, use of resources, retention of providers, and access to care [11]. Therefore, training programs that include purposefully and strategically designed IPE are a critical component in preparing students for today's healthcare settings. The area of infectious diseases, in particular, requires teams of professionals working together to develop effective and timely treatments, practices, and protocols to combat emerging and reemerging diseases.

On a larger scale, collaboration across disciplines and nations is needed in order to solve today's healthcare issues. Shifting population demographics, social change, and global threats to population health demand a diverse, patient-centered, culturally competent, and team-competent healthcare workforce with broad perspectives and the ability to implement inter-disciplinary and interprofessional strategies. Most importantly, we must recognize that new approaches to healthcare practice and education are needed, so that a better future for global health can be attained.

Author Contribution JC and CHV prepared the manuscript.

\section{Declaration}

Conflict of Interest No conflicts of interest

\section{References}

1. Frenk J, Chen L, Bhutta ZA, Cohen J, Crisp N, Evans T, Fineberg H, Garcia P, Ke Y, Kelley P, Kistnasamy B, Meleis A, Naylor D, Pablos-Mendez A, Reddy S, Scrimshaw S, Sepulveda J, Serwadda D, Zurayk H. Health professionals for a new century: transforming education to strengthen health systems in an interdependent world. Lancet. 2010;376(9756):1923-58. https://doi.org/10.1016/ S0140-6736(10)61854-5.

2. Collins FS, Stoffels P. Accelerating COVID-19 therapeutic interventions and vaccines (ACTIV): an unprecedented partnership for unprecedented times. JAMA. 2020;323(24):2455-7. https://doi. org/10.1001/jama.2020.8920.

3. Vega CP, Nyarko E. The Value of Team-Based Management in a Virus Outrbreak. 2020. https://www.medscape.org/viewarticle/ 924696.

4. Hawryluck L, Lapinsky SE, Stewart TE. Clinical review: SARS - lessons in disaster management. Crit Care. 2005;9(4):384-9. https://doi.org/10.1186/cc3041. 
5. Knobler SL, Burroughs T, Mahmoud A, Lemon SM. Ensuring an Infectious Disease Workforce: Education and Training Needs for the 21st Century: Workshop Summary. Board on Global Health: Paper presented at the Forum on Microbial Threats; 2006.

6. Green BN, Johnson CD. Interprofessional collaboration in research, education, and clinical practice: working together for a better future. J Chiropr Educ. 2015;29(1):1-10. https://doi.org/ 10.7899/JCE-14-36.

7. Institute of Medicine (US). Committee on Quality of Health Care in America. In: Crossing the Quality Chasm: A New Health System for the 21st Century. Washington (DC). 2001. https://doi.org/ $10.17226 / 10027$.

8. Bridges DR, Davidson RA, Odegard PS, Maki IV, Tomkowiak J. Interprofessional collaboration: three best practice models of interprofessional education. Med Educ Online. 2011; 16. https:// doi.org/10.3402/meo.v16i0.6035.

9. Foral PA, Anthone JM, Destache CJ, Vivekanandan R, Preheim LC, Gorby GL, Horne JM, Dobronski LA, Syed JJ, Mindru C, Ali MA, Ali KF, Neemann KA, Bittner MJ. Education and Communication in an Interprofessional Antimicrobial Stewardship Program. J Am Osteopath Assoc. 2016;116(9):588-93. https://doi.org/10. 7556/jaoa.2016.116.

10. World Health Organization. Framework for Action on Interprofessional Education and Collaborative Practice. Geneva. 2010.

11. Lemieux-Charles L, McGuire WL. What do we know about health care team effectiveness? A review of the literature. Med Care Res Rev. 2006;63(3):263-300. https://doi.org/10.1177/1077558706287003.

12. Leipzig RM, Hyer K, Ek K, Wallenstein S, Vezina ML, Fairchild $\mathrm{S}$, Cassel CK, Howe JL. Attitudes toward working on interdisciplinary healthcare teams: a comparison by discipline. J Am Geriatr Soc. 2002;50(6):1141-8. https://doi.org/10.1046/j.1532-5415. 2002.50274.x.

13. Stead W, O'Halloran TD, Bernier M, Zimetbaum PJ, Irish J. Using interprofessional education strategies to improve collaborative attitudes among infectious diseases and cardiology physician trainees. Med Teach. 2012;34(7):594-5. https://doi.org/10.3109/ 0142159X.2012.675102.

14. Walton V, Hogden A, Long JC, Johnson JK, Greenfield D. How do interprofessional healthcare teams perceive the benefits and challenges of interdisciplinary ward rounds. J Multidiscip Healthc. 2019;12:1023-32. https://doi.org/10.2147/JMDH.S226330.
15. Medicine GFoliHPEBoGHIo. In: Interprofessional Education for Collaboration: Learning How to Improve Health from Interprofessional Models Across the Continuum of Education to Practice: Workshop Summary. Washington (DC). 2013. https://doi.org/10.17226/13486.

16. Schmitt M, Blue A, Aschenbrener CA, Viggiano TR. Core competencies for interprofessional collaborative practice: reforming health care by transforming health professionals' education. Acad Med. 2011;86(11):1351. https://doi.org/10.1097/ACM.0b013e3182308e39.

17. Kahaleh AA, Danielson J, Franson KL, Nuffer WA, Umland EM. An interprofessional education panel on development, implementation, and assessment strategies. Am J Pharm Educ. 2015;79(6):78. https://doi.org/10.5688/ajpe79678.

18. Guraya SY, Barr H. The effectiveness of interprofessional education in healthcare: a systematic review and meta-analysis. Kaohsiung J Med Sci. 2018;34(3):160-5. https://doi.org/10.1016/j.kjms.2017.12.009.

19. King HE, Battles J, Baker DP, Alonso A, Salas E, Webster J, Toomey L, Salisbury M. TeamSTEPPS ${ }^{\mathrm{TM}}$ : Team Strategies and Tools to Enhance Performance and Patient Safety. In: Henriksen K BJ, Keyes MA, et al., editors. (ed) Advances in Patient Safety: New Directions and Alternative Approaches (Vol. 3: Performance and Tools). vol (Vol. 3: Performance and Tools). 2008.

20. Alexandraki I, Hernandez CA, Torre DM, Chretien KC. Interprofessional Education in the Internal Medicine Clerkship Post-LCME Standard Issuance: Results of a National Survey. J Gen Intern Med. 2017;32(8):871-6. https://doi.org/10.1007/s11606-017-4004-3.

21. Al Achkar M, Hanauer M, Colavecchia C, Seehusen DA. Interprofessional education in graduate medical education: survey study of residency program directors. BMC Med Educ. 2018;18(1):11. https://doi.org/10.1186/s12909-017-1104-z.

22. Ribeiro da Cunha B, Fonseca LP, Calado CRC. Antibiotic Discovery: Where Have We Come from, Where Do We Go? Antibiotics. 2019; 8(2). https://doi.org/10.3390/antibiotics8020045.

23. MacDougall C, Schwartz BS, Kim L, Nanamori M, Shekarchian $\mathrm{S}$, Chin-Hong PV. An Interprofessional Curriculum on Antimicrobial Stewardship Improves Knowledge and Attitudes Toward Appropriate Antimicrobial Use and Collaboration. Open Forum Infectious Diseases. 2016.

Publisher's Note Springer Nature remains neutral with regard to jurisdictional claims in published maps and institutional affiliations. 